\section{ORIGINAL RESEARCH}

\author{
L. Shi \\ D. Wang \\ W.C.W. Chu \\ R.G. Burwell \\ B.J.C. Freeman \\ P.A. Heng \\ J.C.Y. Cheng
}

\title{
Volume-Based Morphometry of Brain MR Images in Adolescent Idiopathic Scoliosis and Healthy Control Subjects
}

\begin{abstract}
BACKGROUND AND PURPOSE: Adolescent idiopathic scoliosis (AIS) is a spinal deformity with unknown cause. Previous studies have suggested that subclinical neurologic abnormalities are associated with AIS. The objective of this prospective study was to characterize systematically neuroanatomic changes in patients with left thoracic AIS vs right thoracic AIS and healthy control subjects by using volumebased morphometry.
\end{abstract}

\begin{abstract}
MATERIALS AND METHODS: Our current study involved 9 girls with left thoracic AIS and 20 girls with right thoracic AIS vs 11 and 17 matched female control subjects, respectively. Voxel-based morphometry (VBM), deformation-based morphometry (DBM), and tensor-based morphometry (TBM) were used to analyze the MR images aligned with a specific brain template of local adolescent girls. The statistical $t$ test was used in VBM and TBM, and the Hotelling $T^{2}$ test was applied in DBM.
\end{abstract}

RESULTS: Using VBM, we found statistically significant differences $(P<.05)$ in the white matter attenuation of the genu of the corpus callosum and left internal capsule (left thoracic AIS $<$ control subjects). In contrast, no significant differences were observed between patients with right thoracic AIS and control subjects.

concLusıons: White matter attenuation in the corpus callosum and left internal capsule, responsible for interhemispheric communication and conduit of the corticothalamic projectional fibers, respectively, were found to be significantly lower in left thoracic AIS compared with control subjects; however, this was not the case in right thoracic AIS. Confirmation of the findings is required in future research, which needs to evaluate the relationship of white matter abnormality to curve laterality, pathogenesis, and prognosis in patients with AIS, with biologic significance and possible therapeutic correction.

A dolescent idiopathic scoliosis (AIS) is a 3D spinal deformity occurring commonly in adolescent girls. The prevalence of AIS can be up to $4 \%$ of a population. ${ }^{1}$ The current treatment of AIS is still unsatisfactory because the etiopathogenesis remains unclear. ${ }^{2}$ To enable evidence-based treatments and better prognostication of curve progression, research on the cause of AIS has been conducted along different paths (eg, bone growth, vertebral growth modulation, neuromotor mechanisms, hormonal system, and genetics)..$^{3-5}$

It has been hypothesized that the cause of AIS could involve subclinical neuromuscular dysfunction in which occult neurologic disorder during growth could present as a deformity in the multisegmented spine. Patients with AIS have been demonstrated to respond poorly on combined tests for visual and proprioceptive function compared with control subjects, ${ }^{6} \mathrm{im}-$ paired spatial orientation, ${ }^{7}$ poor postural control, ${ }^{8-12}$ abnor-

Received December 19, 2008; accepted after revision February 2, 2009.

From the Departments of Diagnostic Radiology and Organ Imaging (L.S., W.C.W.C.), Computer Science and Engineering (D.W., P.A.H.), and Orthopaedics and Traumatology (J.C.Y.C.), Chinese University of Hong Kong, Shatin, New Territories, Hong Kong; Centre for Spinal Studies and Surgery (R.G.B.), Nottingham University Hospitals Trust, Queen's Medical Centre Campus, Nottingham, UK; and Department of Spinal Surgery (B.J.C.F.), Royal Adelaide Hospital, University of Adelaide, North Terrace, Adelaide, Australia.

This work was supported by the British Scoliosis Research Foundation (Project ID: BSRF2006/1-pilot study) and a grant from the Research Grants Council of the Hong Kong Special Administrative Region, China (Project ID: CUHK 4453/06M).

Please address correspondence to Prof. Winnie C.W. Chu, Department of Diagnostic Radiology and Organ Imaging, Chinese University of Hong Kong, Shatin, NT, Hong Kong; e-mail: winnie@med.cuhk.edu.hk

Indicates open access to non-subscribers at www.ajnr.org

DOI 10.3174/ajnr.A1577 mal nystagmus response to caloric testing, ${ }^{13-15}$ asymmetric otolith vestibulo-ocular responses, ${ }^{16}$ and abnormal sway patterns by use of stabilimetry. ${ }^{17}$ Abdominal reflexes were found to be asymmetric by another study. ${ }^{18}$ One study has reported an increase in somatosensory-evoked potential (SSEP) latencies corrected for body height in 68 of 100 patients with AIS. ${ }^{19}$ Our previous research findings have suggested that the site of SSEP abnormality in AIS originated from a level above the cervical spine ${ }^{20}$ and likely to be linked to disturbances along the spinocortical pathway.

These observations of subclinical neurologic abnormalities motivated us to investigate systematically the detailed statistical brain morphometry in patients with AIS. We hypothesized that intracerebral structural abnormalities might be present that could contribute to inappropriate postural adjustment during the growth spurt and so explain the progressive spinal deformity observed in the early adolescence of subjects with AIS.

Available techniques for statistical quantification of the neuroanatomic changes from MR imaging data include voxelbased morphometry (VBM), ${ }^{21}$ deformation-based morphometry (DBM), ${ }^{22}$ and tensor-based morphometry (TBM), ${ }^{23}$ which are named volume-based morphometry in this study, as they perform unbiased measurement and comprehensive analysis of every voxel in the volumetric data, instead of the segmented region of interest. The 3 methods were applied for cross-validation purposes. Volume-based morphometry has been widely used in neuroscience, ${ }^{24}$ neuropsychological, ${ }^{25}$ and biomedical research. In our study, we applied volume-based morphometry in the testing of our 


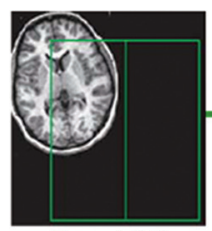

Raw MRI

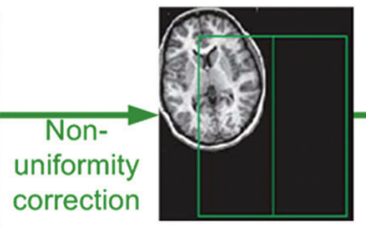

Non-uniformity corrected brain

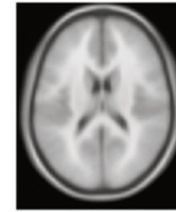

Template brain

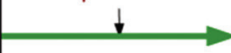

Spatial standardization
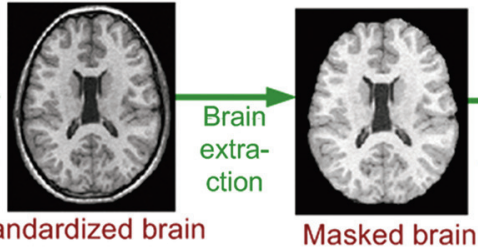

Standardized brain
Tissue classific ation Classified Brain

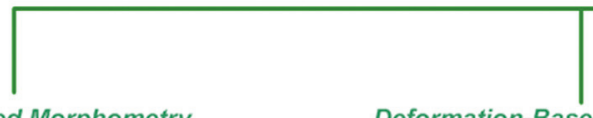

Voxel-Based Morphometry

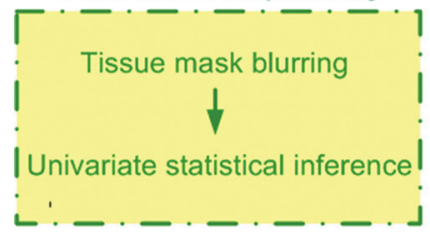

Deformation-Based Morphometry

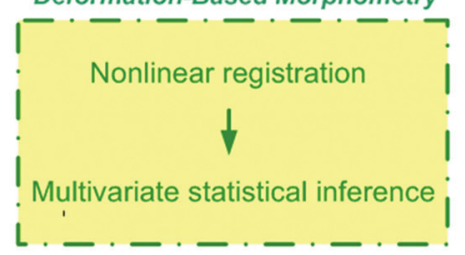

Fig 1. An overview of volume-based morphometry methods

hypothesis that there are neuroanatomic changes in patients with AIS on MR imaging. As the cause of left thoracic AIS (which is the less common type) has been reported to be different from right thoracic AIS (the more common type ${ }^{26}$ and it has been reported that left thoracic curves have a slightly higher prevalence of neuroanatomic abnormalities than right thoracic curves, ${ }^{27}$ patients with left thoracic and right thoracic AIS were analyzed separately in this study.

\section{Materials and Methods}

\section{Subjects and MR Imaging}

In this study, patients with any history of head injury, headache, back injury, weakness or numbness in 1 or more limbs, urinary incontinence, nocturnal enuresis, or any space-occupying lesion found on screening MR imaging were excluded. Also, all subjects were required to have normal results on detailed neurologic examination. A total of 57 right-handed Chinese adolescent girls were prospectively recruited consecutively. Among them, there were 9 girls aged 11 to 16 years (mean age, 14 years) with a left thoracic curve (mean Cobb angle, $19^{\circ}$ ) and 20 girls aged 13 to 17 years (mean age 15) with right-thoracic curve (mean Cobb angle, $33.8^{\circ}$ ). Eleven and 17 age-matched female healthy subjects (as healthy controls for left thoracic AIS and right thoracic AIS respectively) were also recruited to match with the AIS subgroups. The healthy control subjects were invited to participate in the study from local schools. The study was formally approved by the university and hospital ethics committee, and informed written consent was obtained from all subjects and their parents.

For all participants, MR imaging of the brain was performed with a 1.5T MR imaging scanner (Sonata; Siemens, Erlangen, Germany) with use of a quadrature head coil. High-spatial-resolution anatomic imaging was performed with a magnetization-prepared rapid acqui- sition gradient echo sequence. Parameters were TR, 2070 ms; TE, 3.93 ms; TI, $1110 \mathrm{~ms}$; flip angle, $15^{\circ}$; FOV, $230 \mathrm{~mm}$; section thickness, 0.9 mm; gap, none; matrix, $256 \times 256 \times 192$; and NEX, 1 . The sequence yielded high-quality isotropic images with the voxel size of $0.9 \mathrm{~mm} \times$ $0.9 \mathrm{~mm} \times 0.9 \mathrm{~mm}$. The scanning time for each participant was 8.5 minutes.

\section{Data Analysis}

Three volume-based morphometric analysis tools (ie, VBM, ${ }^{21}$ $\mathrm{DBM},{ }^{22}$ and $\mathrm{TBM}^{23}$ ) were applied to quantify the statistical differences among patients with AIS and healthy control subjects. The general pipeline of volume-based morphometry is illustrated in Fig 1.

In our implementation, the common preprocessing procedures for VBM, DBM, and TBM were nonuniformity correction, ${ }^{28}$ spatial standardization, ${ }^{29}$ brain extraction (ie, skull-stripping), ${ }^{30}$ and tissue classification. ${ }^{31}$ Thereafter, the white matter (WM), gray matter (GM), and CSF were analyzed separately. We analyzed the morphologic features of each tissue type by performing the statistical analysis to localize the group differences.

Construction of a population-specific brain template. In volume-based morphometry, brain template plays an important role. The brain template is a reference through which each individual brain volume is mapped to a common space and compared in a voxelwise fashion. More importantly, the deformation field for both DBM and TBM analyses will be calculated from the nonlinear registration between each individual MR imaging and this representative template. Because of the anatomic difference between Chinese brains and Caucasian brains, ${ }^{32-35}$ a population-specific, enhanced brain template was constructed on the basis of brain scans of 101 Hong Kong adolescent girls including 56 patients with right thoracic AIS, 10 patients with left thoracic AIS, and 35 healthy control subjects. 


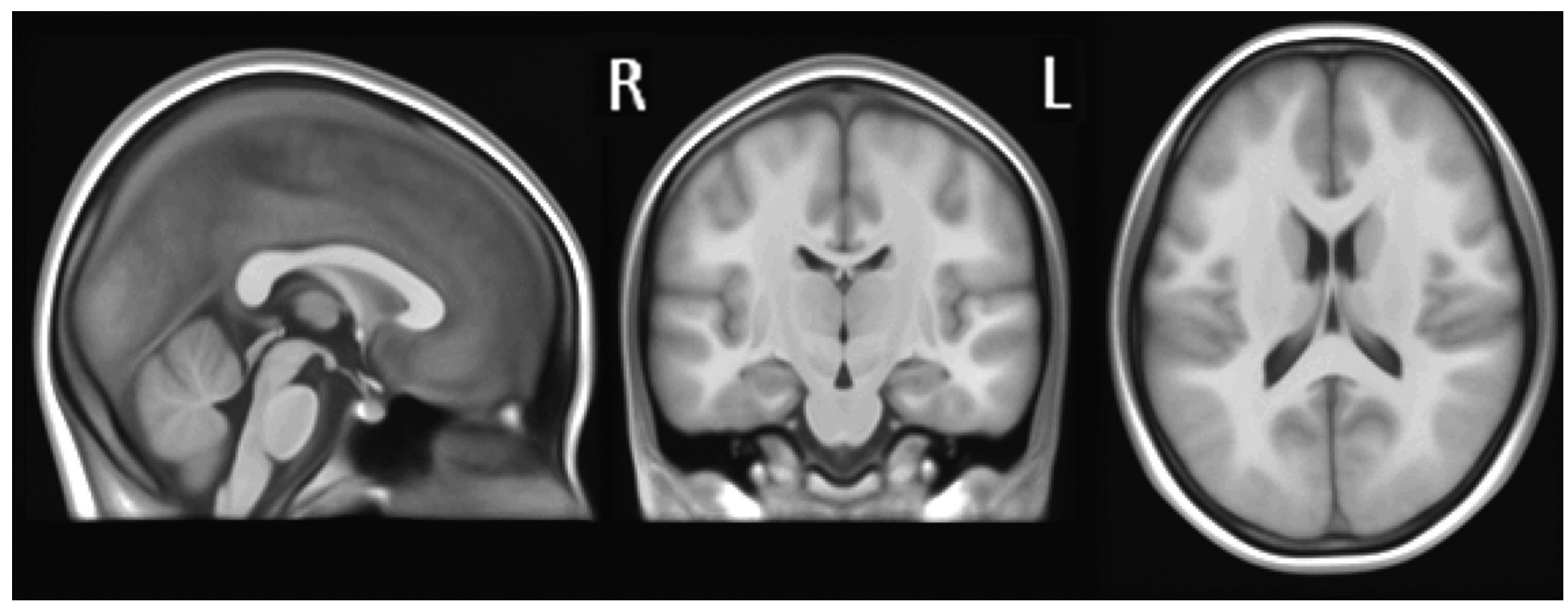

Fig 2. Statistical brain template of 101 Hong Kong adolescents.

In the construction of the population-specific brain template, a coarse-to-fine mechanism of the diffeomorphism of nonlinear registration was used in an iterative manner. Six iterations were used to construct this template. The nonlinear registration was formulated as an optimization problem. Suppose the deformation filed is $\vec{h}$, the template image is $T(\vec{x})$, and the study image is $S(\vec{x})$. Let the Jacobian field of $\vec{h}$ be denoted as $J(\vec{h})$. The energy function we minimized was formulated as follows:

$$
D=\int_{\Omega}|T(\vec{x}-\vec{h})-S(\vec{x})|^{2} d \vec{x}+\lambda \int_{\Omega}|J(\vec{h}) \vec{x}|^{2} d \vec{x},
$$

where $\lambda>0$ is the Lagrange multiplier that controls the trade-off between the 2 terms. In this objective function, the first term requires the local intensities in the study image to be as close as possible to the template image, whereas the second term restricts the stretching of the displacement field by limiting the magnitude of the Jacobian map at each location.

Voxel-based morphometry. According to the central limit theorem, ${ }^{36}$ the data are more normally distributed after Gaussian smoothing. Therefore, the mask of each tissue type was blurred by convolving with an isotropic Gaussian kernel with a user-specified kernel size, which resulted in the attenuation maps of the 3 tissue types.

On the basis of the attenuation map of each tissue type, the $t$ test was applied to produce a map of statistical difference values for the local concentration of each tissue type between patients and control subjects. The critical threshold $t$ that corresponded to $P$ value equating a certain threshold was calculated on the basis of the random field theory. ${ }^{37}$

Deformation-based morphometry. The deformation fields resulting from warping each brain volume to the template were calculated by use of nonlinear registration. Each displacement vector started from the voxel in the individual volume and ended at the corresponding voxel in the template volume, where the correspondence was determined by nonlinear registration.

In the deformation field, because a displacement vector with the length of 3 was associated with each voxel representing the displacement along the 3 directions $x, y$, and $z$, the multivariate statistic was applied. In particular, we applied the Hotelling $T^{2}$ test to evaluate the statistical difference in the deformation field.
Tensor-based morphometry. Tensor-based morphometry aims at localizing regional differences between groups of brains by examining the Jacobian matrix of each and every displacement vector that maps the point in the template image to the corresponding point in the individual image. We calculated the Jacobian matrix by taking the gradients at each element of the displacement vector, which represented the tensor describing relative positions of the neighboring elements. The determinant of the Jacobian matrix is usually used to represent the local volume changes. Statistical analysis of these determinant fields was used to compare the changes of the patient group relative to the healthy control subjects, where a determinant of the Jacobian matrix being greater than 1 indicated the local expansion and a determinant smaller than 1 indicated the local shrinkage. Because only the determinants of the Jacobian matrix were considered, the statistical inference was similar to that used in VBM (ie, univariate statistics).

\section{Results}

\section{A Population-Specific Brain Template}

A population-specific brain template was built on the basis of the MR imaging brain volumes of 101 Hong Kong adolescent girls. The resultant template of the whole brain is shown in Fig 2, which had enhanced properties: the edges around the anatomic structures with small variability across the population were sharpened, and the structures with large group variability, such as the sulci and gyrus, were reasonably blurred.

\section{Results from VBM, DBM, and TBM on Patients with Left Thoracic AIS vs Healthy Control Subjects}

In the first analysis, brain MR imaging data of 9 patients with left thoracic AIS were compared with those of 11 healthy control subjects. With use of VBM, significant group differences (control subjects $>$ patients with AIS) in the "attenuation" of white matter in the genu of the corpus callosum and the left internal capsule (anterior arm) were observed (Fig 3). The results of DBM and TBM revealed a similar pattern of spatial distribution of the statistical values, which, however, did not reach the level of significance (ie, $P<.05$ ). 


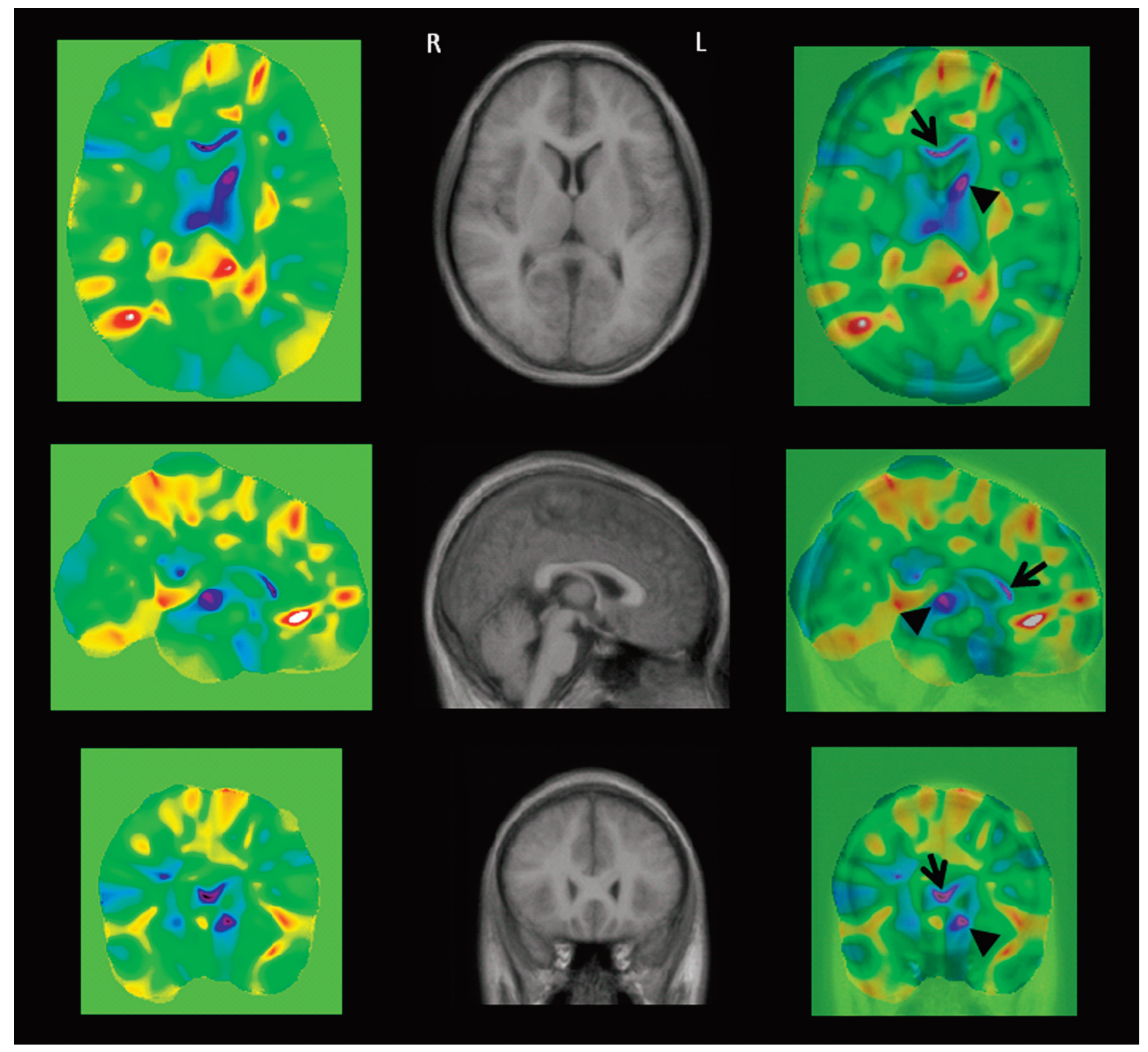

Fig 3. Statistical difference map for white matter densities comparing those in patients with left thoracic AlS vs healthy control subjects. The first column is the statistical $t$ map between the 2 groups. The second column is the averaged structural subjects in this study. The third column is the superimposed images of the first column onto the second column, for better appreciation of the anatomic locations of the statistical changes. The first to third rows correspond to brain sections in the axial, sagittal, and coronal planes, respectively. The purple color highlights regions of significant statistical difference, which include the genu of the corpus callosum (arrows) and the left internal capsule (arrowheads).

\section{Results from VBM, DBM, and TBM on Patients with Right Thoracic AIS vs Healthy Control Subjects}

When comparing brain MR imaging data of 20 patients with right thoracic AIS with those of 17 healthy control subjects, no significant group difference was observed in the tissue attenuation of both gray matter and white matter with VBM, DBM, and TBM.

\section{Discussion}

VBM, DBM, and TBM are 3 typical methods to document the statistical morphometry of the brain. Our study used these volume-based statistical morphometry tools to analyze brain abnormality in patients with AIS.

The principles and implications of the results of VBM, DBM, and TBM are not identical: VBM reveals group differences in "tissue densities" on the basis of the linearly aligned volumetric data. DBM searches for differences in interindividual "positional variations" by comparing the deformation fields calculated from the nonlinear registration, which makes the reliability of the DBM results depend on the performance of the nonlinear registration algorithm. TBM was designed to detect the "local changes" throughout the volumetric data and, similar to DBM, also depends on the performance of nonlinear registration algorithm. However, the nonlinear registration, in general, is a local minimization process, which implies that the resulted mapping would not be unique. It turned out that VBM, DBM, and TBM all resulted in statistic maps with similar patterns of spatial distribution, though only VBM revealed significant findings, which may be the result of the above-mentioned limitation of DBM and TBM. The purpose of applying all these 3 methods in the study was for cross-validation. 
The experience gained from our study in applying VBM, DBM, and TBM in AIS brain morphometry analysis points to the importance of several steps in the volume-based morphometry methods necessary to assure the validity of the analysis:

1. The registration should be accurate and robust. First, a representative template should be adopted. In our implementation, we constructed a brain template by using high-quality MR imaging data of 101 adolescent subjects, including 56 patients with right thoracic AIS, 10 with left thoracic AIS, and 35 healthy control subjects. This template was able to represent the anatomy of the population we analyzed. Second, it is preferred to use the same scanner and scanning sequence to avoid systematic differences in image contrast and noise, as was used in our data acquisition. Third, cases of severe brain structural abnormality such as stroke and tumor should be excluded. The data used in our analysis were acquired from subjects with no obvious brain damage or pathologic condition.

2. The segmentation should be reliable. First, the brain should be correctly separated from the whole head; we guaranteed this in our study by visually checking segmentation results. Second, the image contrast should be high enough so that partial volume artifacts can be minimized. The data we used had adequate image contrast. Third, the image quality should be guaranteed (eg, MR imaging data with a ring effect caused by motion during scanning or subjects with a dental brace should be avoided). Unqualified subjects were excluded in our analysis.

3. The statistics should be performed correctly. First, the size of the smoothing kernel should match the expected effect size. The size of the Gaussian kernel in our analysis was set to 10 , which is suitable for brain analysis. Moreover, multiple comparison correction should be performed, which was achieved by use of random field theory in our analysis.

The less common left thoracic AIS is more often associated with neuroanatomic abnormalities vs right thoracic AIS. ${ }^{27,38}$ In our study, girls with left thoracic AIS showed white matter attenuation in the genu of the corpus callosum, the principal commissural fiber bundle connecting the left and right hemispheres and the anterior limb of the left internal capsule, to be significantly lower than normal. A similar difference was not found in patients with right thoracic AIS. This finding is important for AIS, a disorder of bilateral (left-right or mirrorimage) symmetry. ${ }^{26}$ Abnormal function of the corpus callosum might affect the coordination of the left and right sides of the body, while the internal capsule carries projectional fibers between the cerebral cortex and the thalamus. The thalamus is the relay station for the limbic, sensory, and motor functional systems. The etiologic question is whether the detected abnormalities of the corpus callosum and internal capsule have pathogenetic significance (ie, a primary causative feature of left thoracic AIS) or are secondary to motor imbalance or inappropriate postural adjustment. Additional investigation is needed to establish whether these abnormalities are related to the laterality of other AIS curves, thoracolumbar and lumbar, and to infratentorial neuroanatomic abnormalities, particularly for children younger than 11 years. ${ }^{38}$ A larger sample and a longitudinal study are required to establish whether 1) the brain abnormalities correct or persist with age and 2) whether they are predictive of curve progression. These and other questions need to be addressed in additional human and animal studies.

\section{Conclusion}

Volume-based morphometry analysis is a useful tool to systematically investigate the neuroanatomic changes in AIS. In girls with left, but not right, thoracic AIS, this morphometric study with MR imaging showed the white matter attenuation in the corpus callosum and left internal capsule to be significantly lower compared with healthy control subjects. Confirmation is required in future research to evaluate the relationship of the white matter abnormality to curve laterality, pathogenesis, and prognosis in patients with AIS, with biologic significance and possible therapeutic correction along this direction.

\section{Acknowledgment}

We thank Prof. Tomáš Paus, director of the Brain \& Body Centre, University of Nottingham, UK, for his valuable advices before and during preparation of this manuscript.

\section{References}

1. Weinstein S. Adolescent idiopathic scoliosis: natural history. In: Weinstein SL ed. The Pediatric Spine: Principles and Practice. Philadelphia: Lippincott Williams \& Wilkins; 2001:355-70

2. Miller N. Cause and natural history of adolescent idiopathic scoliosis. Orthop Clin North Am 1999;30:343-52

3. Burwell RG, Dangerfield PH, Freeman BJ. Concepts on the pathogenesis of adolescent idiopathic scoliosis. Bone growth and mass, vertebral column, spinal cord, brain, skull, extra-spinal left-right skeletal length asymmetries, disproportions and molecular pathogenesis. Stud Health Technol Inform 2008; $135: 3-52$

4. Ward K, Nelson LM, Chettier R, et al. Genetic profile predicts curve progression in adolescent idiopathic scoliosis. In: Scoliosis Research Society 43rd Annual Meeting and Course, Salt Lake City, Utah, September 10-13, 2008:59

5. Kouwenhoven JW, Castelein RM. The pathogenesis of adolescent idiopathic scoliosis: review of the literature. Spine 2008;33:2898-908

6. Yekutiel M, Robin GC, Yarom R. Proprioceptive function in children with adolescent idiopathic scoliosis. Spine 1981;6:560-66

7. Herman R, Mixon J, Fisher A, et al. Idiopathic scoliosis and the central nervous system: a motor control problem. The Harrington lecture, 1983. Scoliosis Res Soc Spine 1985;10:1-14

8. Adler N, Bleck EE, Rinsky LA, et al. Balance reactions and eye-hand coordination in idiopathic scoliosis. J Orthop Res 1986;4:102-07

9. Byl NN, Gray JM. Complex balance reactions in different sensory conditions: adolescents with and without idiopathic scoliosis. J Orthop Res 1993;11: 215-27

10. Byl NN, Holland S, Jurek A, et al. Postural imbalance and vibratory sensitivity in patients with idiopathic scoliosis: implications for treatment. J Orthop Sports Phys Ther 1997;26:60-68

11. Gauchard GC, Lascombes P, Kuhnast M, et al. Influence of different types of progressive idiopathic scoliosis on static and dynamic postural control. Spine 2001;26:1052-58

12. Beaulieu M, Toulotte C, Gatto L, et al. Postural imbalance in non-treated adolescent idiopathic scoliosis at different periods of progression. Eur Spine J 2009; $18: 38-44$

13. O’Beirne J, Goldberg C, Dowling FE, et al. Equilibrial dysfunction in scoliosiscause or effect? J Spinal Disord 1989;2:184-89

14. Sahlstrand T, Petruson B. A study of labyrinthine function in patients with adolescent idiopathic scoliosis. I. An electro-nystagmographic study. Acta Orthop Scand 1979;50:759-69

15. Yamada K, Yamamoto H, Nakagawa Y, et al. Etiology of idiopathic scoliosis. Clin Orthop Relat Res 1984;50-57

16. Wiener-Vacher SR, Mazda K. Asymmetric otolith vestibulo-ocular responses in children with idiopathic scoliosis. J Pediatr 1998;132:1028-32

17. Lidström J, Friberg S, Lindström L, et al. Postural control in siblings to scoliosis patients and scoliosis patients. Spine 1988;13:1070-74

18. Zadeh HG, Sakka SA, Powell MP, et al. Absent superficial abdominal reflexes 
in children with scoliosis. An early indicator of syringomyeli. J Bone Joint Surg Br 1995;77:762-67

19. Hausmann ON, Boni T, Pfirrmann CW, et al. Preoperative radiological and electrophysiological evaluation in 100 adolescent idiopathic scoliosis patients. Eur Spine Jl 2003;12:501-06

20. Chau WW, Guo X, Fu LL, et al. Abnormal somatosensory evoked potential (SSEP) in adolescents with idiopathic scoliosis - the site of abnormality. In: Sawatzky BJ, ed. International Research Society of Spinal Deformities Symposium. Vancouver, Canada; 2004:279-81

21. Ashburner J, Friston K. Voxel-based morphometry-the methods. Neuroimage 2000;11:805-21

22. Ashburner J, Hutton C, Frackowiak R, et al. Identifying global anatomical differences: Deformation-based morphometry. Hum Brain Mapp 1998;6: $348-57$

23. Ashburner J, Friston KJ. Morphometry. In: Frackowiak RSJ, Friston KJ, Frithet CD, eds. Human Brain Function. London: Academic Press; 2003

24. Paus T, Zijdenbos A, Worsley K, et al. Structural maturation of neural pathways in children and adolescents: in vivo study. Science 1999;283:1908-11

25. Maguire E, Gadian D, Johnsrude I, et al. Navigation-related structural change in the hippocampi of taxi drivers. Proc Natl Acad Sci U S A 2000;97:4398-403

26. Burwell R, Dangerfield P, Freeman B, et al. Etiologic theories of idiopathic scoliosis: the breaking of bilateral symmetry in relation to left-right asymmetry of internal organs, right thoracic adolescent idiopathic scoliosis (AIS) and vertebrate evolution. Stud Health Technol Inform 2006;123:385-90

27. Goldberg CJ, Moore DP, Fogarty EE, et al. Left thoracic curve patterns and their association with disease. Spine 1999;24:1228-33
28. Sled J, Zijdenbos A, Evans A. A nonparametric method for automatic correction of intensity nonuniformity in MRI data. IEEE Tran Med Imaging, 1998;17:87-97

29. Collins D, Neelin P, Peters T, et al. Automatic 3-D intersubject registration of MR volumetric data in standardized Talairach space. J Comp Assist Tomogr 1994;18:192-205

30. Smith S. Fast robust automated brain extraction. Human Brain Mapp 2002;17:143-55

31. Cocosco C, Zijdenbos A, Evans A. A fully automatic and robust brain MRI tissue classification method. Med Image Analysis 2003;7:513-27

32. Chi TK, Chang C. The sulcal pattern of the Chinese brain. Am J Phys Anthropol 1941;28:167-211

33. Tsai SH. A study of sulcal pattern of Chinese brain. J Med Sci 1977;2:596-601

34. Shellshear JL. The brain of the Aboriginal Australian. A study in cerebral morphology. Philos Trans Royal Soc London, Series B, Biol Sci 1937;227:293-409

35. Evans A, Collins D, Neelin P, et al. Three-dimensional correlative imaging: applications in human brain mapping. Functional Neuroimaging: Technical Foundations 1994;145-62

36. Feller W. The fundamental limit theorems in probability. Bull Am Math Soc 1945;51:800-32

37. Worsley K, Marrett S, Neelin P, et al. A unified statistical approach for determining significant signals in images of cerebral activation. Human Brain Mapp 1996;4:58-73

38. Maenza RA. Juvenile and adolescent idiopathic scoliosis: magnetic resonance imaging evaluation and clinical indications. J Pediatr Orthop B 2003;12:295302 